\title{
Randomised controlled trial of non-directive counselling, cognitive-behaviour therapy, and usual general practitioner care for patients with depression. II: Cost effectiveness
}

Peter Bower, Sarah Byford, Bonnie Sibbald, Elaine Ward, Michael King, Margaret Lloyd, Mark Gabbay

\begin{abstract}
Objective To compare the cost effectiveness of general practitioner care and two general practice based psychological therapies for depressed patients. Design Prospective, controlled trial with randomised and patient preference allocation arms.

Setting General practices in London and greater Manchester.

Participants 464 of 627 patients presenting with depression or mixed anxiety and depression were suitable for inclusion.

Interventions Usual general practitioner care or up to 12 sessions of non-directive counselling or cognitive-behaviour therapy provided by therapists.

Main outcome measures Beck depression inventory scores, EuroQol measure of health related quality of life, direct treatment and non-treatment costs, and cost of lost production.

Results 197 patients were randomly assigned to treatment, 137 chose their treatment, and 130 were randomised only between the two psychological therapies. At four months, both non-directive counselling and cognitive-behaviour therapy reduced depressive symptoms to a significantly greater extent than usual general practitioner care. There was no significant difference in outcome between treatments at 12 months. There were no significant differences in direct costs, production losses, or societal costs between the three treatments at either four or 12 months. Sensitivity analyses did not suggest that the results depended on particular assumptions in the statistical analysis.

Conclusions Within the constraints of available power, the data suggest that both brief psychological therapies may be significantly more cost effective than usual care in the short term, as benefit was gained with no significant difference in cost. There are no significant differences between treatments in either outcomes or costs at 12 months.
\end{abstract}

\section{Introduction}

The clinical effectiveness of psychological therapy in primary care has received increasing study in recent years. ${ }^{1}$ Although several trials have reported use of health services or included an economic component, ${ }^{2-5}$ full economic analyses of psychological therapies in primary care are relatively rare.

The present study concerns the cost effectiveness of non-directive counselling, cognitive-behaviour therapy, and routine general practitioner care in the management of depression and mixed anxiety and depression. Their comparative cost effectiveness has received attention in one previous trial. ${ }^{6}$ Although only counselling produced significantly better clinical outcomes compared with general practitioner care, the patients in that group were less severely ill at baseline, which made interpretation difficult. The limited costing methodology found no difference between therapies in total cost: both were more than twice as expensive as general practitioner care.

\section{Participants and methods}

This economic analysis was based on a randomised controlled trial of three treatments for depressed patients in primary care, and methodological details of the trial are reported in full in the accompanying paper. It was designed as a cost effectiveness study, with the Beck depression inventory ${ }^{7}$ as the main outcome and the EuroQol ${ }^{8}$ as a secondary outcome measure. A societal perspective was taken, which included direct treatment costs, direct non-treatment costs, and costs of lost production.

General practitioners referred 627 depressed patients, of whom 464 were eligible for entry into the study. The main sample consisted of patients randomly allocated to one of the three treatments $(n=197)$. Patients who were unwilling to accept random allocation were offered the option of choosing their preferred treatment $(n=137)$ or being randomised between the two psychological therapies only $(n=130)$. A full comparison of patients allocated by randomisation or preference will be reported elsewhere; the focus of the current analysis is on the main sample of 197 patients. However, in sensitivity analysis, we combined the sample of patients randomised between the two psychological therapies with those randomised to the therapies using conventional randomisation in order to increase the sample size available for analysis of cost differences between the therapies.

Patients allocated to psychological therapy were offered 6-12 sessions with a qualified therapist. Patients in usual care were managed by their general practitioner.

\section{Cost data}

Direct treatment costs included contacts with primary and secondary public health services, psychotropic drugs, and private health services. We collected data on use of resources from two sources. We searched general practice medical records for the 12 months before and after referral to our study in order to collect information on general practitioner and practice nurse consultations, hospital referrals, and use of psychotropic drugs. We also gathered details from patients'
Editorial by Briggs

National Primary Care Research and Development Centre (NPCRDC), University of Manchester Peter Bower research fellow Bonnie Sibbald professor

Centre for Health Economics, York University, York YO10 5DD

Sarah Byford research fellow

Department of Psychiatry and Behavioural Sciences, Royal Free and University College Medical School, University College London, London NW3 2PF

Elaine Ward research fellow Michael King head

Margaret Lloyd reader

Department of Primary Care, University of Liverpool Mark Gabbay senior lecturer Correspondence to: M King m.king@rfc. ucl.ac.uk

BMJ 2000;321:1389-92 
Table 1 Resources used 12 months after entry to trial by patients given usual general practitioner care or one of two psychological therapies for depression. Values are mean (SD) number of contacts unless stated otherwise

\begin{tabular}{llcc} 
& \multicolumn{3}{c}{ Use of resources (1 year) } \\
\cline { 2 - 4 } Service & $\begin{array}{c}\text { Usual GP care } \\
(\mathbf{n}=\mathbf{6 7})\end{array}$ & $\begin{array}{c}\text { Cognitive-behaviour } \\
\text { therapy ( } \mathbf{n = 6 3 )}\end{array}$ & $\begin{array}{c}\text { Non-directive } \\
\text { counselling ( } \mathbf{n}=67)\end{array}$ \\
\hline Primary care services & & & \\
\hline GP surgery contacts: & $9.12(5.1)$ & $6.48(4.6)$ & $7.71(6.6)$ \\
\hline Non-attendances & $0.29(0.66)$ & $0.26(0.55)$ & $0.34(0.81)$ \\
\hline Out of hours contacts & $0.02(0.12)$ & $0.03(0.13)$ & $0.02(0.06)$ \\
\hline GP cooperative & $0.16(1.1)$ & $0.07(0.25)$ & $0.07(0.30)$ \\
\hline GP home visits & $0.05(0.27)$ & $0.03(0.18)$ & $0.04(0.27)$ \\
\hline Practice nurse contacts: & $0.53(1.1)$ & $0.69(0.95)$ & $0.41(0.68)$ \\
\hline Non-attendances & $0.02(0.12)$ & $0.03(0.18)$ & $0.002(0.01)$ \\
\hline Protocol therapy† & & & \\
\hline Sessions attended: & NA & $4.97(3.5)$ & $6.44(4.2)$ \\
\hline Non-attendances & NA & $1.36(1.5)$ & $0.97(1.1)$ \\
\hline
\end{tabular}

\section{Medication‡}

No (\%) of patients taking drug:

\begin{tabular}{lccc}
\hline Antidepressants & $33(49)$ & $17(27)$ & $20(30)$ \\
\hline Minor tranquillisers & $12(18)$ & $4(6)$ & $10(15)$ \\
\hline$\beta$ blockers & $3(4)$ & $5(8)$ & $2(3)$ \\
\hline Major tranquillisers & 0 & 0 & $1(1)$ \\
\hline \begin{tabular}{l} 
Specialist services§ \\
\hline $\begin{array}{l}\text { Mental health referrals (including } \\
\text { therapy based in primary care) }\end{array}$
\end{tabular} & $0.52(0.88)$ & $0.22(0.52)$ & $0.25(0.59)$ \\
\hline Non-psychiatric referrals & $0.93(1.28)$ & $0.92(1.26)$ & $0.93(1.13)$
\end{tabular}

$\mathrm{GP}=$ general practitioner. $\mathrm{NA}=$ not applicable.

*Unit costs from Personal Social Services Research Unit. ${ }^{9}$

†Unit costs from trial service.

fUnit costs from British National Formulary. ${ }^{11}$

$\S U$ it costs from Chartered Institute of Public Finance and Accountancy ${ }^{10}$ for secondary services and from

trial service for primary care (in one case a London trial therapist was provided free from a local trust, but a cost equal to the average cost for London was applied).

self reports at baseline and at four and 12 months follow up; details included visits to health professionals, hospital referrals, and use of prescribed drugs. Two psychologists (PB and $\mathrm{EW}$ ) and a general practitioner (MG) collected data. No test of the reliability of the data extraction was undertaken.

Direct non-treatment costs included costs of child care and travel. Only four patients, however, reported child care costs, and so these were ignored. Although we asked about travel costs to secondary care, data were not reported by a substantial number of patients who had such visits recorded in their notes. Thus we also ignored these costs and included only the travel costs of visits to primary care professionals and to the psychological therapy sessions.

For indirect costs, we calculated the cost of production losses from information gathered by face to face or postal interviews. Data included employment status, weeks worked, current wage rate, and an estimate of time lost from work through illness.

We determined unit costs for the financial year 1997-8 from a variety of sources, including the databases of the Personal Social Services Research Unit $^{9}$ and of the Chartered Institute of Public Finance and Accountancy ${ }^{10}$ and the British National Formulary. ${ }^{11}$ We based travel costs on either self reported fares or mileage (with a unit cost supplied by the Automobile Association). The cost of time off work was based on self reported annual, monthly, or weekly pay before tax.

Full medical records were available for searches for 364 patients $(78 \%)$; we used a combination of incomplete notes (such as incomplete temporary patient notes) and self reporting for 39 patients (8\%); and we used patient self reports only for 61 patients
(13\%). We imputed missing data for general practice and practice nurse consultations, wages, time off work, and travel using the mean from the relevant treatment group and site (London or Manchester). Missing prescribing and referral data were not imputed and were assumed to be zero. We completed partial data (such as missing drug dose) according to various decision rules (such as clinical judgment regarding commonly prescribed doses for drugs).

\section{Statistical methods}

There was no power calculation for costs; we calculated the sample size on the basis of expected clinical outcomes. All analyses were carried out on an intention to treat basis. Although costs were not normally distributed, we compared mean costs using standard $t$ tests and analysis of variance methods, and confirmed the validity of results using bootstrapping. ${ }^{12} 13$ This approach allows inferences to be made about the arithmetic mean, ${ }^{14}$ which is not possible with logarithmic transformation or conventional nonparametric tests. The primary analysis was of total costs, but we also give details of use of individual resource components (such as primary care, protocol therapy). The primary analysis was of total costs incurred in the 12 months after the baseline measurements, but we also adjusted results for the total cost of care in the 12 months before entry into the study using multiple regression. We conducted sensitivity analyses to assess the robustness of results to changes in assumptions. Discounting was unnecessary as neither costs nor benefits were recorded beyond 12 months.

\section{Results}

\section{Clinical outcome}

Full details on clinical outcomes can be found in the accompanying paper. Briefly, patients in all three arms of the trial improved on the primary outcome measure, but the patients in both psychological therapy groups made significantly greater clinical gains in the first four months after allocation. However, all groups had equivalent outcomes at 12 months. There were no significant differences in outcome between the three groups in terms of the EuroQol.

\section{Costs}

Table 1 gives details of resource use over 12 months. Patients given usual general practitioner care recorded more consultations, greater use of antidepressant drugs, and more psychiatric referrals. Table 2 lists the total costs in each psychological therapy group as compared with usual general practitioner care at four and 12 months.

We found no significant differences between the three groups at four months in total societal costs (analysis of variance $\mathrm{F}=0.508, \mathrm{df}=196, \mathrm{P}=0.60$ ), total direct care costs $(\mathrm{F}=0.176, \mathrm{df}=196, \mathrm{P}=0.83)$, or total production losses $(\mathrm{F}=0.384, \quad \mathrm{df}=196, \quad \mathrm{P}=0.68)$. Equally, we found no significant difference between the groups at 12 months in total societal costs $(\mathrm{F}=0.449$, $\mathrm{df}=196, \mathrm{P}=0.63)$, total direct care costs $(\mathrm{F}=0.111$, $\mathrm{df}=196, \quad \mathrm{P}=0.89), \quad$ or total production losses $(\mathrm{F}=0.374, \mathrm{df}=196, \mathrm{P}=0.68)$. These results did not differ when adjusted for costs before entry into the study. In no case did the bootstrap results suggest that the use of the $t$ test was invalid. 
Table 2 Total costs $(£)$ for usual general practitioner care and two psychological therapies for depression at four and 12 months follow up

\begin{tabular}{|c|c|c|c|c|c|c|c|c|}
\hline \multirow[b]{2}{*}{ Resource use } & \multicolumn{2}{|c|}{ Usual GP care } & \multicolumn{3}{|c|}{ Cognitive-behaviour therapy } & \multicolumn{3}{|c|}{ Non-directive counselling } \\
\hline & Mean (SD) & $\begin{array}{c}\% \text { of total } \\
\text { societal } \\
\text { cost }\end{array}$ & Mean (SD) & $\begin{array}{c}\% \text { of total } \\
\text { societal } \\
\text { cost }\end{array}$ & $\begin{array}{c}\text { Mean }(95 \% \mathrm{CI}) \text { difference } \\
\text { from GP care }\end{array}$ & Mean (SD) & $\begin{array}{c}\% \text { of total } \\
\text { societal } \\
\text { cost }\end{array}$ & $\begin{array}{c}\text { Mean }(95 \% \mathrm{Cl}) \text { difference } \\
\text { from GP care }\end{array}$ \\
\hline \multicolumn{9}{|l|}{ Direct costs: } \\
\hline Primary care & $64.5(73.0)$ & 10.3 & $39.7(27.4)$ & 7.9 & 24.8 (5.9 to 43.9$)$ & $37.7(26.2)$ & 5.4 & 26.8 (8.0 to 45.7$)$ \\
\hline Drugs & $13.1(26.9)$ & 2.1 & $5.2(16.5)$ & 1.0 & 7.9 (0.3 to 15.7$)$ & $6.6(18.3)$ & 1.0 & $6.5(-1.3$ to 14.4$)$ \\
\hline Outpatient services & $98.1(237.8)$ & 15.6 & $32.1(66.9)$ & 6.4 & 66.0 (5.8 to 126.3) & $23.9(51.6)$ & 3.4 & $74.2(14.9$ to 133.4$)$ \\
\hline Inpatient services & $65.6(372.5)$ & 10.5 & $1.8(14.5)$ & 0.4 & $63.8(-27.2$ to 154.7$)$ & $39.3(297.6)$ & 5.6 & $26.3(-89.0$ to 141.5$)$ \\
\hline Protocol therapy & 0 & 0 & $133.3(71.8)$ & 26.6 & $-133.3(-151.4$ to -115.3$)$ & $143.5(72.0)$ & 20.4 & $-143.5(-161.0$ to -125.9$)$ \\
\hline Travel costs & $2.6(4.5)$ & 0.4 & $3.5(5.9)$ & 0.7 & $-0.9(-2.7$ to 1.0$)$ & $6.5(9.6)$ & 0.9 & $-3.9(-6.4$ to -1.3$)$ \\
\hline Total & $244.0(597.5)$ & 38.9 & $215.5(108.6)$ & 43.0 & $28.5(-119.6$ to 176.6$)$ & $257.5(356.7)$ & 36.7 & $-13.5(-181.7$ to 154.7$)$ \\
\hline Total indirect costs & $383.7(1194.3)$ & 61.1 & $286.1(701.3)$ & 57.0 & 97.6 (-245.1 to 440.25$)$ & $444.4(1127.2)$ & 63.3 & -60.7 (-457.6 to 336.1$)$ \\
\hline Total societal costs & $627.7(1359.8)$ & 100 & $501.6(715.3)$ & 100 & 126.1 (-254.5 to 506.7$)$ & $701.9(1228.4)$ & 100 & -74.2 (-517.1 to 368.6$)$ \\
\hline \multicolumn{9}{|l|}{ Costs at 12 months } \\
\hline \multicolumn{9}{|l|}{ Direct costs: } \\
\hline Drugs & $40.7(77.8)$ & 3.3 & $12.2(37.5)$ & 1.2 & 28.5 (7.5 to 49.6$)$ & $24.1(54.2)$ & 1.7 & 16.6 (-6.4 to 39.5$)$ \\
\hline Outpatient services & $201.2(344.9)$ & 16.5 & $105.9(251.9)$ & 10.0 & $95.3(-9.2$ to 199.7$)$ & $90.3(175.8)$ & 6.5 & 110.9 (17.0 to 204.7) \\
\hline Inpatient services & $107.6(425.1)$ & 8.8 & $74.2(381.8)$ & 7.0 & $33.4(-107.1$ to 174.0$)$ & $106.6(428.6)$ & 7.6 & $1.0(-144.9$ to 146.9$)$ \\
\hline Protocol therapy & 0 & 0 & $164.3(104.1)$ & 15.5 & $-164.3(-190.6$ to -138.1$)$ & $171.2(97.1)$ & 12.2 & $-171.2(-194.9$ to -147.5$)$ \\
\hline $\begin{array}{c}\text { Travel costs } \\
\end{array}$ & $4.8(7.8)$ & 0.4 & $5.5(8.8)$ & 0.5 & $-0.7(-3.6$ to 2.2$)$ & $10.7(17.8)$ & 0.8 & $-5.9(-10.6$ to -1.2$)$ \\
\hline Direct costs & $472.9(779.3)$ & 38.8 & $448.9(471.6)$ & 42.3 & 24.0 (-201.3 to 249.3) & $501.4(614.8)$ & 35.9 & $-28.5(-268.4$ to 211.3$)$ \\
\hline Indirect costs & $744.7(1796.4)$ & 61.2 & $611.6(1370.4)$ & 57.7 & 133.1 (-424.0 to 690.2$)$ & $897.2(2336.1)$ & 64.2 & -152.5 (-864.7 to 559.6$)$ \\
\hline Total societal costs & $1217.5(2013.0)$ & 100 & $1060.5(1471.1)$ & 100 & 157 (-458.0 to 772.2$)$ & $1398.6(2474.1)$ & 100 & -181.1 (-951.9 to 589.7) \\
\hline
\end{tabular}

$\mathrm{GP}=$ general practitioner.

\section{Sensitivity analyses}

To evaluate the robustness and generalisability of the results, we performed several univariate sensitivity analyses (table 3). These included using full costs for missed appointments in primary care and specialist facilities (thus assuming that the clinicians were unable to fill the time with alternative activities); use of the most expensive alternative drugs (compared with the generic drug used in the initial calculation); use of the national reported average wage instead of self reported wages; and restricting the analysis to patients with full data $(n=170)$. The differences in total cost between the three groups remained non-significant.

In a cost comparison of the two psychological therapies only, we also calculated costs on the basis of all randomised patients (those randomised between the three treatments plus those randomised between the two psychological therapies only, $\mathrm{n}=265$ ). However, there were still no significant differences in costs.

\section{Discussion}

We found no statistically significant differences between the three treatments in total societal costs, direct costs, or the cost of production losses. The overall results are consistent with other recently published studies. ${ }^{5}{ }^{15}$ However, as with clinical outcomes,${ }^{16}$ our finding of no difference in costs must be interpreted with caution. As is usual, cost data were highly variable, and our study may have been underpowered to detect differences in costs that would be considered important by decision makers. However, in our comparisons of patients allocated to the psychological therapies by either of the randomisation procedures we effectively doubled the sample size available and still found no significant differences. Furthermore, none of the sensitivity analyses significantly influenced the results. The validity of our finding of "no difference" may be strengthened through further original research with larger samples of patients, although recruitment in primary care is often difficult ${ }^{17}$ and the costs of such studies may prove problematic. Meta-analysis of cost data from similar studies may be another method of overcoming this problem.

Our three treatment groups showed little variation in the use of specialist services for non-psychiatric problems (table 1). The differences between the groups were in the use of primary care services, psychotropic drugs, and mental health facilities. To assess the impact of the psychological therapies on the use of all other healthcare services, we removed the cost of the two therapies from the analysis. At four months, there was a significant difference in direct treatment costs

Table 3 Sensitivity analyses for one year costs for usual general practitioner care and two psychological therapies for depression

\begin{tabular}{|c|c|c|c|c|}
\hline \multirow[b]{2}{*}{ Costs analysed } & \multicolumn{3}{|c|}{ Mean (SD) costs $(£)$} & \multirow[b]{2}{*}{$\begin{array}{c}\text { Analysis of } \\
\text { variance }\end{array}$} \\
\hline & $\begin{array}{l}\text { Usual GP care } \\
\qquad(n=67)\end{array}$ & $\begin{array}{c}\text { Cognitive- } \\
\text { behaviour therapy } \\
(n=63)\end{array}$ & $\begin{array}{l}\text { Non-directive } \\
\text { counselling } \\
(n=67)\end{array}$ & \\
\hline \multicolumn{5}{|l|}{ Societal costs } \\
\hline Main analysis & $1217.5(2013.0)$ & $1060.5(1471.1)$ & $1398.6(2474.1)$ & $P=0.63$ \\
\hline Non-attendances included & $1227.4(2014.3)$ & $1072.4(1471.9)$ & $1415.1(2475.9)$ & $P=0.63$ \\
\hline Most expensive alternative drug & $1218.7(2015.0)$ & 1060.7 (1471.2) & $1398.8(2474.1)$ & $P=0.64$ \\
\hline National wage rate & $2526.5(3042.0)$ & $2034.8(2629.3)$ & $2750.9(4155.3)$ & $P=0.46$ \\
\hline Complete data & $1202.8(2075.7)$ & $954.9(1047.2)$ & $1409.6(2595.2)$ & $P=0.49$ \\
\hline \multicolumn{5}{|l|}{ Direct costs } \\
\hline Main analysis & $472.9(779.3)$ & $448.9(471.6)$ & $501.4(614.8)$ & $P=0.89$ \\
\hline Missed sessions included & $482.7(783.5)$ & $460.8(473.8)$ & $517.9(641.1)$ & $P=0.88$ \\
\hline Most expensive alternative drug & $474.0(784.9)$ & $449.1(471.6)$ & $501.6(614.9)$ & $P=0.90$ \\
\hline Complete data & $419.9(484.4)$ & $470.5(498.9)$ & $532.3(654.6)$ & $P=0.55$ \\
\hline \multicolumn{5}{|l|}{ Indirect costs } \\
\hline Main analysis & $744.7(1796.4)$ & $611.6(1370.4)$ & $897.2(2336.1)$ & $P=0.68$ \\
\hline National wage rate & $2053.6(2730.6)$ & $1585.9(2551.4)$ & $2249.5(4012.4)$ & $P=0.48$ \\
\hline Complete data* & $782.9(1923.3)$ & $484.4(908.5)$ & $877.3(2442.2)$ & $P=0.52$ \\
\hline
\end{tabular}

$\mathrm{GP}=$ general practitioner.

${ }^{*}$ No of patients with complete data: usual GP care $=57$; cognitive-behaviour therapy $=55$; non-directive counselling $=58$. 


\section{What is already known on this topic}

The cost effectiveness of psychological therapies in general practice for depression is not always measured in randomised clinical trials

A small number of published trials have reported that the costs of psychological therapy and general practitioner care are similar

\section{What this study adds}

The data suggest that both brief psychological therapies may be significantly more cost effective than usual general practitioner care in the short term, as benefit was gained with no significant difference in cost

This finding must be considered preliminary, given the low power of the cost calculations

There was no evidence that psychological therapies were more cost effective than usual care in the long term

between the group given usual general practitioner care and those given cognitive-behaviour therapy (mean difference $£ 163$ (95\% confidence interval £12 to $£ 313) ; \mathrm{P}=0.031$ ). This suggests that in the short term the costs of providing cognitive-behaviour therapy were recouped through reduced use of other healthcare services. There were no such significant differences between general practitioner care and non-directive counselling at four months, nor between general practitioner care and either therapy at 12 months.

None of the three treatments seemed to be associated with markedly lower rates of time off work or lost production costs. The inclusion of production losses in economic evaluation is still a matter for debate, mainly because of criticisms of the valuation methods used. ${ }^{18}$ The valuation of production losses on the basis of earnings, as used in this study, ignores the fact that the existence of unemployment allows the replacement of workers who leave the labour force at little cost. Hence, attention has recently turned to the friction cost method of calculation, which attempts to account for the level of scarcity in the labour market. ${ }^{19}$ Although we did not try the friction cost method, we know that these costs would lie somewhere between the human capital valuations we reported and zero. Since the conclusions of our study were not altered by inclusion or exclusion of productivity costs from the analysis, friction cost valuations would not affect the results. Equally controversial is the method by which zero value was placed on productivity losses for patients not in paid employment. However, the more equitable analysis (using an average wage rate for all patients) did not significantly influence the results.

We excluded various cost elements from the analysis-such as the travel costs associated with specialist referrals and the costs of non-psychotropic drugs-and assumed missing data on referrals and drugs to be zero because of the considerable resources that would be required to collect such data. Thus the calculated total direct costs are probably lower than the actual costs incurred. The impact of these exclusions, however, is likely to be small. Travel costs were a relatively small proportion of total costs and differed little between the three groups at the final follow up.
The number of patients with missing data was relatively low, and the sensitivity analysis provided no evidence that this was a significant influence on the results. It is unlikely that the inclusion of such costs would significantly change our results.

In conclusion, the use of psychological therapies in general practice was associated with short term benefits in the mental health of depressed patients compared with usual general practitioner care. Since our study failed to find a significant difference in total costs between the three interventions it is possible that the psychological therapies were also more cost effective than usual care in the short term. However, this finding must be considered preliminary, given the low power of the cost calculations. At 12 months, we found no significant differences between the three treatments in outcomes or total costs, and thus there was no evidence that psychological therapies were more cost effective than usual care in the long term. Given such equivalence, commissioners of services are in a position to decide on services based on factors other than outcomes and costs, such as staff and patient preferences or staff availability.

Contributors: MK, BS, and ML conceived the idea for the trial, obtained research funding, and supervised the conduct of the trial and data collection. EW, ML, PB, and MG undertook recruitment of practices and patients and conducted the data management. SB and PB analysed the data. All authors contributed to the writing of the paper. MK is the guarantor for the study.

Funding: The study was supported by a grant from the NHS Executive Health Technology Assessment Programme. The views expressed in this paper are those of the authors only and are not attributable to the Department of Health.

Competing interests: None declared.

1 Roth A, Fonagy P. What works for whom? A critical review of psychotherapy research. London: Guildford, 1996.

2 Boot D, Gillies P, Fenelon J, Reubin R, Wilkins M, Gray P. Evaluation of the short-term impact of counseling in general practice. Patient Educ Counsel 1994;24:79-89.

Gournay K, Brooking J. The community psychiatric nurse in primary care: an economic analysis. J Adv Nurs 1995;22:769-78.

4 Robson M, France R, Bland M. Clinical psychologist in primary care: controlled clinical and economic evaluation. BMJ 1984;288:1805-8.

5 Harvey I, Nelson S, Lyons R, Unwin C, Monaghan S, Peters T. A randomized controlled trial and economic evaluation of counselling in primary care. Br J Gen Pract 1998;48:1043-8.

6 Scott A, Freeman C. Edinburgh primary care depression study: treatment outcome, patient satisfaction, and cost after 16 weeks. BMJ 1992;304:883-7.

7 Beck A, Steer R. Beck depression inventory: manual. San Antonio: Psychological Corporation, 1987.

8 EuroQol Copyright Group. EuroQol-a new facility for the measurement of health related quality of life. Health Policy 1990;16:199-208.

9 Netten A, Dennet J, Knight J. Unit costs of health and social care. Canterbury: University of Kent, Personal Social Services Research Unit, 1998.

10 Chartered Institute of Public Finance and Accountability. The health service financial database 1997. London: Chartered Institute of Public Finance and Accountability; 1997.

11 British Medical Association, Royal Pharmaceutical Society of Great Britain. British national formulary. London: BMA, RPS, 1998. (No 35.)

12 Efron B, Tibshirani R. An introduction to the bootstrap. New York: Chapman and Hall, 1993.

13 Briggs A, Gray A. Handling uncertainty when performing economic evaluation of health care interventions. Health Technol Assess 1999;3(2).

14 Barber J, Thompson S. Analysis and interpretation of cost data in randomised controlled trials: review of published studies. BMJ 1998;317:1195-200.

15 Friedli K, King M, Lloyd M. The economics of employing a counsellor in general practice: analysis of data from a randomised controlled trial. $\mathrm{BrJ}$ Gen Pract 2000;50:276-83.

16 Altman D, Bland M. Absence of evidence is not evidence of absence. BMJ $1995 ; 311: 485$.

17 Fairhurst K, Dowrick C. Problems with recruitment in a randomised controlled trial of counselling in general practice: causes and implications. J Health Serv Res Policy 1996;1:77-80.

18 Johnston K, Buxton M, Jones D, Fitzpatrick R. Assessing the costs of healthcare technologies in clinical trials. Health Technol Assess 1999;3(6).

19 Koopmanschap M, Rutten F. A practical guide for calculating indirect costs of disease. Pharmacoeconomics 1996;10:460-6.

(Accepted 10 August 2000) 